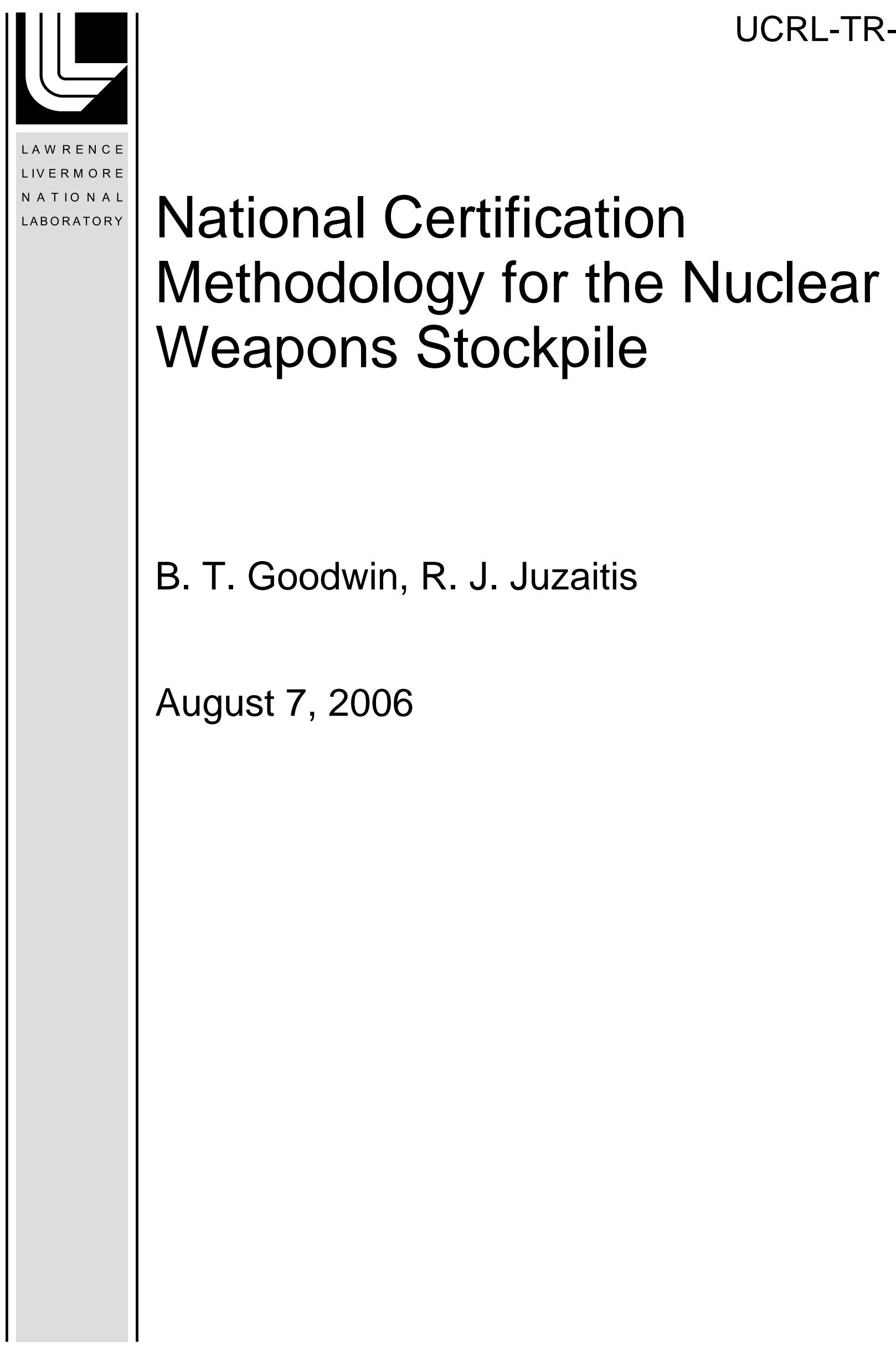


This document was prepared as an account of work sponsored by an agency of the United States Government. Neither the United States Government nor the University of California nor any of their employees, makes any warranty, express or implied, or assumes any legal liability or responsibility for the accuracy, completeness, or usefulness of any information, apparatus, product, or process disclosed, or represents that its use would not infringe privately owned rights. Reference herein to any specific commercial product, process, or service by trade name, trademark, manufacturer, or otherwise, does not necessarily constitute or imply its endorsement, recommendation, or favoring by the United States Government or the University of California. The views and opinions of authors expressed herein do not necessarily state or reflect those of the United States Government or the University of California, and shall not be used for advertising or product endorsement purposes.

This work was performed under the auspices of the U.S. Department of Energy by University of California, Lawrence Livermore National Laboratory under contract No. W-7405-Eng-48. 


\title{
National Certification Methodology for the Nuclear Weapon Stockpile
}

\author{
by \\ Bruce T. Goodwin, Associate Director for Defense and Nuclear Technologies, LLNL \\ and \\ Raymond J. Juzaitis, Associate Director for Weapon Physics, LANL
}

\section{Executive Summary}

Lawrence Livermore and Los Alamos National Laboratories have developed a common framework and key elements of a national certification methodology called Quantification of Margins and Uncertainties (QMU). A spectrum from senior managers to weapons designers has been engaged in this activity at the two laboratories for on the order of a year to codify this methodology in an overarching and integrated paper. Following is the certification paper that has evolved. In the process of writing this paper, an important outcome has been the realization that a joint Livermore/Los Alamos workshop on QMU, focusing on clearly identifying and quantifying differences between approaches between the two labs plus developing an even stronger technical foundation on methodology, will be valuable. Later in FY03, such a joint laboratory workshop will be held. One of the outcomes of this workshop will be a new version of this certification paper.

A comprehensive approach to certification must include specification of problem scope, development of system baseline models, formulation of standards of performance assessment, and effective procedures for peer review and documentation. This document concentrates on the assessment and peer review aspects of the problem. In addressing these points, a central role is played by a "watch list" for weapons derived from credible failure modes and performance gate analyses. The watch list must reflect our best assessment of factors that are critical to weapons performance. High fidelity experiments and calculations as well as full exploitation of archival test data are essential to this process. Peer review, advisory groups and red teams play an important role in confirming the validity of the watch list. The framework for certification developed by the Laboratories has many basic features in common, but some significant differences in the detailed technical implementation of the overall methodology remain. Joint certification workshops held in June and December of 2001 and continued in 2002 have proven useful in developing the methodology, and future workshops should prove useful in further refining this framework. Each laboratory developed an approach to certification with some differences in detailed implementation. The general methodology introduces specific quantitative indicators for assessing confidence in our nuclear weapon stockpile. The quantitative indicators are based upon performance margins for key operating characteristics and components of the system, and these are compared to uncertainties in these factors. These criteria can be summarized in a quantitative metric (for each such characteristic) expressed as: 


$$
\text { Confidence Ratio }(\mathrm{CR})=\text { Margin / Uncertainty, } \quad \text { where } \mathrm{CR}>1
$$

(i.e., confidence in warhead performance depends upon CR significantly exceeding unity for all these characteristics).

These Confidence Ratios are proposed as a basis for guiding technical and programmatic decisions on stockpile actions. This methodology already has been deployed in certifying weapons undergoing current life extension programs or component remanufacture. The overall approach is an adaptation of standard engineering practice and lends itself to rigorous, quantitative, and explicit criteria for judging the robustness of weapon system and component performance at a detailed level. There are, of course, a number of approaches for assessing these Confidence Ratios.

The general certification methodology was publicly presented for the first time to a meeting of Strategic Command SAG in January 2002 and met with general approval. At that meeting, the Laboratories committed to further refine and develop the methodology through the implementation process. This paper reflects the refinement and additional development to date. There will be even further refinement at a joint laboratory workshop later in FY03.

A common certification methodology enables us to engage in peer reviews and evaluate nuclear weapon systems on the basis of explicit and objective metrics. The clarity provided by such metrics enables each laboratory and our common customers to understand the meaning and logic of technical and management decisions affecting stockpile performance and safety.

\section{Stockpile Stewardship}

The United States Government (USG) entered a moratorium on nuclear testing in 1992 and since then has been maintaining its current nuclear weapon stockpile without nuclear testing. Additionally, the USG has not yet required that any weapons of fundamentally new design be developed for the stockpile. These decisions changed the nature of the weapons program from a program that designs, tests and deploys weapons to a program that determines, to a high degree of confidence, when an existing or refurbished weapon design will continue to perform as intended. For most of the history of the US nuclear weapons program, nuclear testing was a critical component for stockpile weapon certification. The codes used in the design and certification process employed models that, in many cases, were incomplete or inaccurate representations of relevant physical processes. The quantity and quality of calculations that could be run were also constrained by computing hardware limitations. As a result, computational models often employed ad-hoc multipliers to match data from Above Ground Experiments (AGEX) or nuclear tests. These computational models worked best for limited interpolation between nuclear tests. However, throughout the nuclear testing era, computational capability, 
AGEX facilities, and our ability to diagnose both nuclear and non-nuclear experiments continued to evolve.

We find ourselves in a different world today. For more than half a decade, we have been developing the tools required to conduct stockpile stewardship in the post nuclear test era. We are developing and applying enhanced predictive capabilities that incorporate improved theoretical, computational and experimental capabilities needed for certification without conducting nuclear tests. Advances in AGEX technology are making possible more precise measurements of some of the detailed physics that occurs during the operation of a nuclear weapon. Improved physics models plus the enormous increases in supercomputer speed and capacity brought about by the Accelerated Strategic Computing Initiative (ASCI) enable the use of very high resolution (both spatially and temporally), high-fidelity code calculations. Such calculations will enable us to reduce our reliance on ad-hoc normalization factors. The ASCI codes, validated against AGEX and archival nuclear test data, have strong potential to improve our confidence in extrapolation (in addition to interpolation) from past nuclear test results. The application of these new stewardship tools to Stockpile Life Extension Programs (SLEPs) and other stockpile issues requires a certification methodology that is quantitative, rigorous and transparent to external review.

\section{Rigorous Certification Standards}

Certification is the process, culminating in a formal declaration by the Laboratory Directors, that establishes that nuclear weapons meet Military Characteristics (MCs) and Stockpile-to-Target-Sequence (STS) requirements. The certification methodology must include a rigorous set of quantitative standards. These quantitative standards must ensure that, when met, there are adequate margins against credible failure modes, or equivalently, that design parameters remain within their performance gates. Some degradation in expected performance of stockpiled weapons, either from recently recognized original design or manufacturing flaws ("birth defects") or changes arising from aging (observed, e.g., through surveillance) or remanufacture, can be anticipated. Certification may be viewed as a process wherein design parameters, performance gates, uncertainties, and margins are evaluated against one another to determine whether the functional requirements of the nuclear warhead have been satisfied. Certification thus becomes a positive action based on quantitative evaluations of weapons performance as measured against explicit standards.

In a "Performance Gate" analysis (LANL), we view a nuclear weapon as a physical system whose time evolution defines a performance or functional requirement timeline. The system evolution is punctuated by a number of critical points that represent key events and separate the timeline into a number of natural stages of operation (see Figure 1). Performance gates are assigned to physical variables characterizing device behavior at each critical design point. The lower and upper boundaries of the performance gates represent the range of variation in the physical variables for which an expert can supply convincing evidence that device performance will, with high confidence, meet MCs and STS requirements. Within the performance gates, a design point typically assumes a 
range of values (the designed operating range), and this entire range is ideally located a safe distance from the gate boundaries to ensure that the device operates properly (see Figure 2). The gates may be evaluated in terms of confidence factors or in terms of probability that the device metrics fall within acceptable limits. Typically gates will be evaluated in the context of potential failure mechanisms and with regard to possible changes from tested configurations.

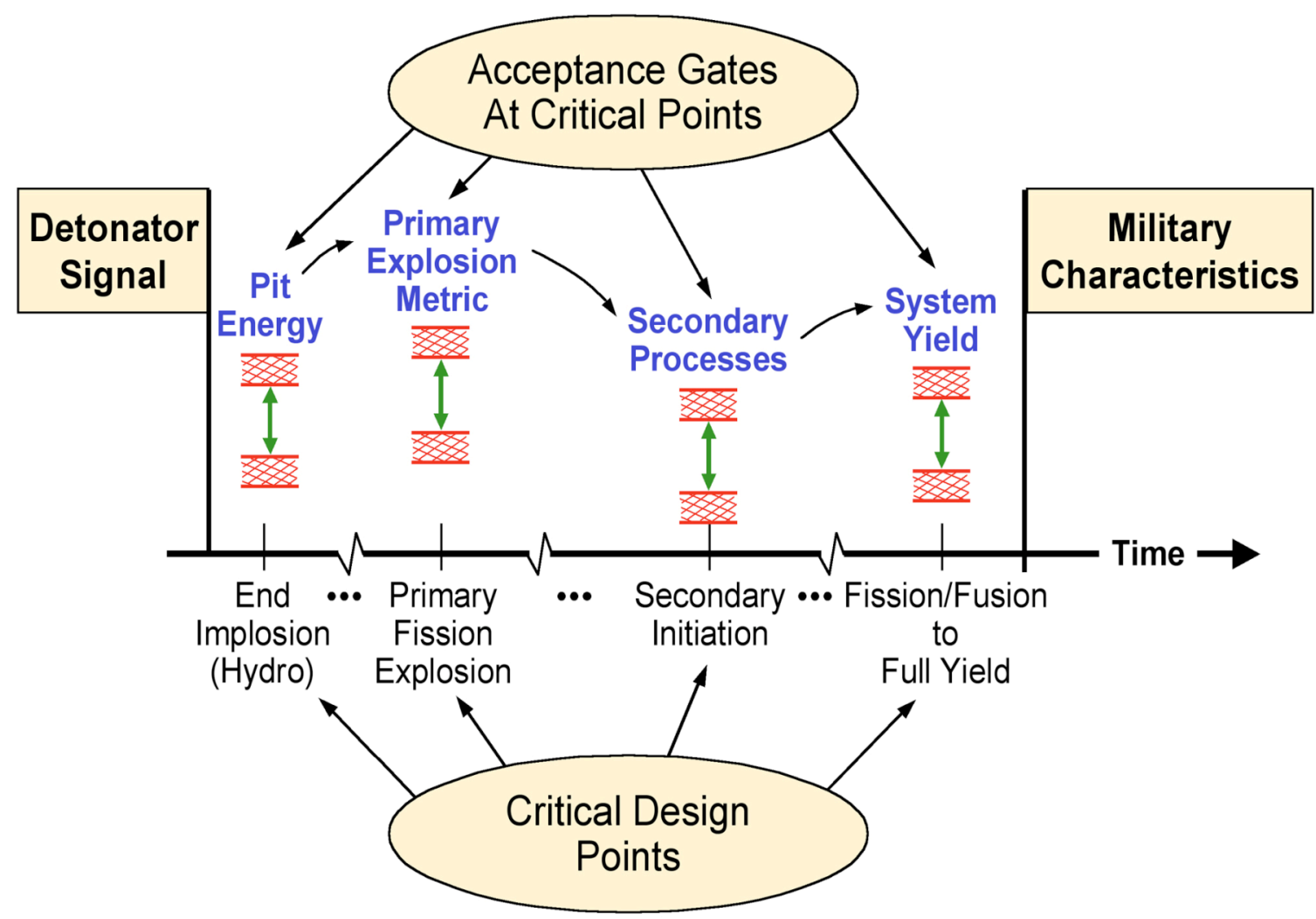

Figure 1: Critical points during system evolution 


\section{Typical Performance Gate}

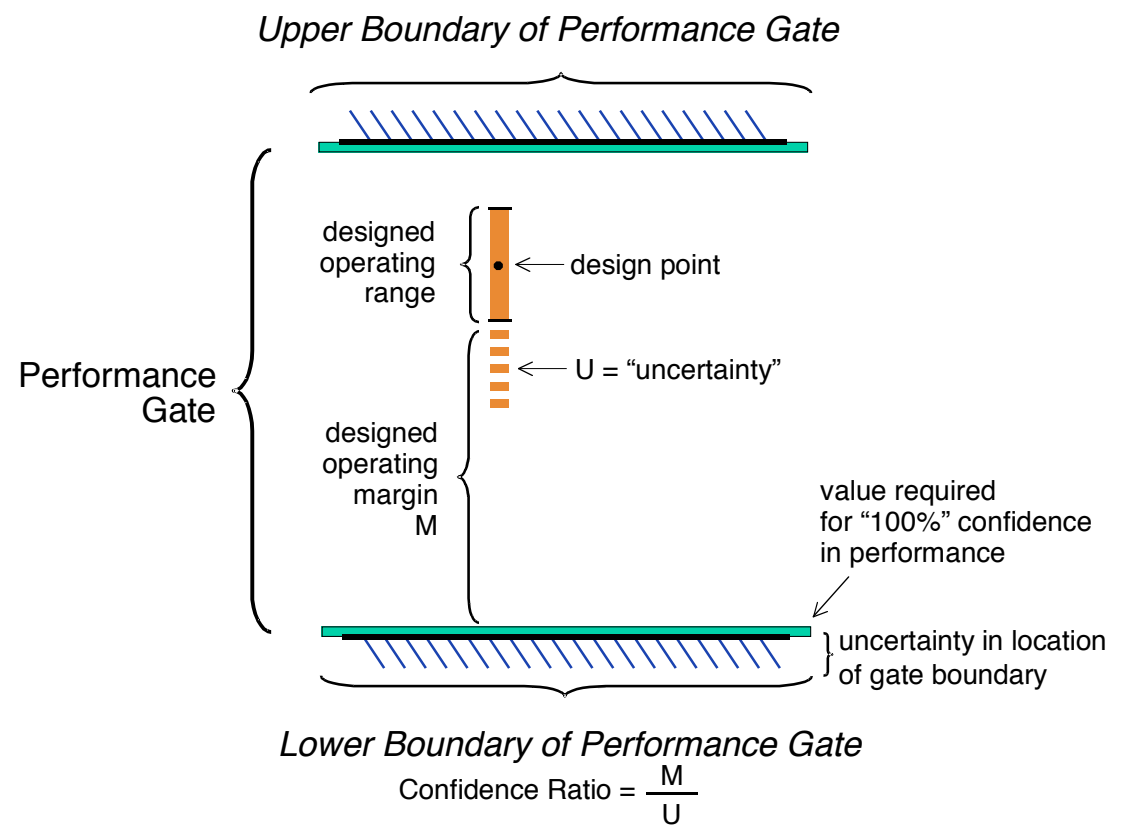

Figure 2: An illustration of a design point within a performance gate and how the Confidence Ratio is defined.

In dealing with potential failure modes for current weapons (LLNL), we must address a broad range of issues. Typical issues include: engineering features introduced during life extension programs, performance in extreme STS environments, performance under aged conditions, marginal performance, degradation of key materials, surveillance replacement pits, high explosive (HE) degradation and cracking, detonator deterioration, detonator redesign, metal corrosion, and proposed changes to manufacturing processes. How then can we have confidence that we have systematically addressed the credible failure modes for device and engineering issues? To do this, we assemble a group of experts who identify critical stages in device evolution, establish metrics to ensure proper performance, and identify a list of credible failure modes and performance issues for the current stockpile. This group then works down the functional operation "tree" for a nuclear weapon to develop a watch list of items that constitutes the observables of credible failure modes (see Figure 3). In general, this must be an on-going process.

Having developed the watch list, margins and associated uncertainties must then be quantified for all of the items. The quantitative results of this process enable the responsible individuals to prioritize the watch list and thereby make rational decisions about the allocations of program resources to stockpile needs. Key to this certification strategy is that margin must always significantly exceed uncertainty for all critical issues. We therefore establish our standard for changes to be a Confidence Ratio (CR) defined as the ratio of the margin to the sum of salient uncertainties, where $C R>1$. Figure 4 shows an example of the performance gate approach as applied to system components. 


\section{A group of experts identified a list of credible failure}

modes and issues for the current stockpile

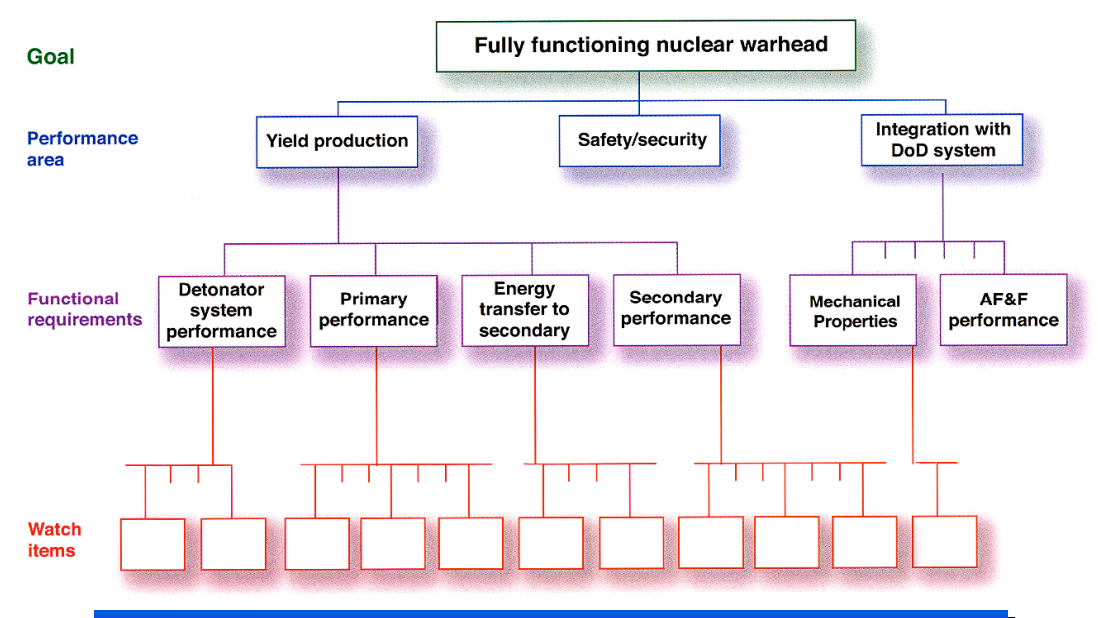

This should be a continuous process

Figure 3: Weapon function "tree" used to identify watch list items.

In both "Performance Gate" and "Failure Mode" analyses, margins against known failure modes give us the tools to manage system risks in light of uncertainties in our knowledge. Confidence Ratios can be used to determine the relative ranking of risks and set program priorities. They can also be used to determine when research efforts have reached their goals. In this way, confidence factors (or ratios) can bring transparency and closure to program elements.

Our approach defines the strategy for certification and for the stockpile stewardship program. Certification must be based on rigorous, quantitative standards for each stage of device function and for all credible failure modes. Maintaining design parameters within their gates and margins with Confidence Ratio $>1$ for each credible failure mode is necessary for proper performance throughout device evolution. The ultimate limit (and goal) of this certification methodology is to confidently quantify the range of credible yields of any aged or rebuilt primary or secondary and the minimum required drive for any aged or rebuilt secondary to within the uncertainty limits established by nuclear test experience. Thus, the certification methodology, leads to specific goals. It provides closure. 


\section{Margins against known failure modes help us manage system risks in}

light of known and unknown uncertainties in our knowledge

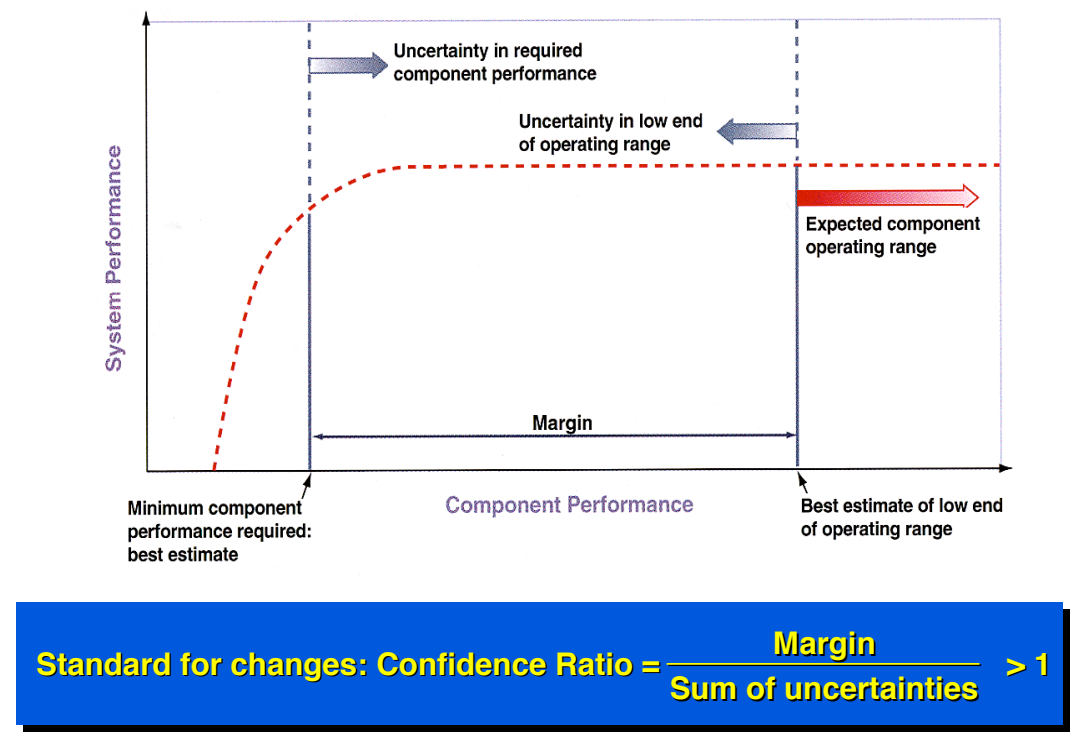

Figure 4: Margin is the excess performance beyond the minimum need to successfully

function and the minimum performance allowed under normal operation. The uncertainties are associated with our incomplete knowledge of the limits of margin. The Confidence Ratio $(\mathrm{CR})$ then becomes $\mathrm{CR}=$ margin $/ \Sigma$ uncertainty and $\mathrm{CR}>1$ is always required to maintain confidence.

\section{Experience with System Application}

QMU either has been, or is being, applied to several weapon systems in the stockpile. During the certification process for a recent warhead refurbishment program, a diverse team of weapon experts assembled to develop a failure modes "watch list." Confidence ratios were proposed for all critical elements. The refurbishment team (not the team who developed the watch list) paid particular attention to nuclear performance over the full range of stockpile-to-target-sequence environments. Our experience with QMU in this refurbishment has led us to apply the approach to a current engineering development program for the refurbishment of a second warhead type. Performance gates are being used in major SLEPs to help prioritize expensive experiments. For example, a decision for a recent major AGEX experiment was based on the relevance of the performance gate it addressed and a quantitative assessment of the potential increase in confidence through anticipated reduction in uncertainty.

In these system applications, how can we ensure that all credible failure modes and/or performance gates have been considered? Clearly, known issues must be addressed up front. It is easiest to deal with what one knows about. Further, we must continue efforts to address past nuclear test surprises in order to convert these surprises to known issues. The most daunting issues, however, are those that one suspects exist but does not yet know about, the unknown unknowns. Open and critical evaluation is essential to 
covering all salient issues. Peer review is essential. External advisory panels can contribute a distinct perspective. Finally, "red teams" can increase the level of confidence in the QMU procedure. A "red team" is an entirely separate team empowered to examine and question all aspects of the design team's work. They can initiate and do their own work (unlike a peer review team) and can pursue alternate paths. Taking multiple approaches to the review process will help us to achieve an understanding of failure modes and their remediation that is as complete as possible in the absence of further nuclear tests.

\section{Conclusions and Path Forward}

The stockpile refurbishment schedule for this new decade is demanding and will require many complex capabilities. Complicating this demanding schedule is the fact that history indicates that we should expect a major stockpile "surprise" every few years. We must be aware that this schedule, combined with finite resources, has delayed development of required certification capabilities relative to the pace of stockpile needs. This underscores the need for a methodology that allows margins to be managed against uncertainties in order to understand the state of the stockpile and so rationally make priority decisions. We believe that the certification methodology outlined here is a substantial step toward meeting this need.

We plan, through a series of both local and joint workshops, to continue to expand and refine our certification methodology, and we foresee periodic updates in the formal documentation of the details of QMU implementation. 